
\title{
R Research Soure \\ Development of A Modified Extended Model-Based Fault Detection And Diagnosis Approach
}

\author{
Alireza Alikhani ( $\nabla$ aalikhani@ari.ac.ir) \\ Aerospace Research Institute https://orcid.org/0000-0001-6450-7802 \\ Ghasem Sharifi \\ KNTU: KN Toosi University of Technology
}

Original Article

Keywords: Fault detection, Spacecraft, Reliability, Actuator, Model-based, Applicable

Posted Date: July 8th, 2021

DOI: https://doi.org/10.21203/rs.3.rs-652350/v1

License: (9) This work is licensed under a Creative Commons Attribution 4.0 International License.

Read Full License 


\title{
Development of a Modified Extended Model-Based Fault Detection and Diagnosis Approach
}

\author{
Alireza Alikhani ${ }^{1 *}$ \\ , Ghasem Sharifi ${ }^{2}$ \\ ${ }^{1}$ A\&S Research Institute, Ministry of Science, Research and Technology, Tehran, Iran \\ ${ }^{2}$ Department of the Aerospace Engineering, K.N. Toosi University of \\ Technology, Tehran, Iran
}

\begin{abstract}
A supervisory system for space missions is critical due to the high risk of missions, the costs, and the impossibility of adding redundancy. The model-based fault detection approaches are of interest due to their highly responsive speed, robustness against disturbances and uncertainties, and accuracy. Conventional model-based methods have some drawbacks such as feasibility and applicability. In this paper, a modified extended multiple models adaptive estimation (MEMMAE) method is developed which keep both the advantages of the previous model-based methods and take into account some limitations of that. This approach can be performed on various systems to detect and diagnose faults, with appropriate response speed and resistance to uncertainty and disturbances. By combining the recursive least-square algorithm with the extended multiple model adaptive estimation (EMMAE) method, the limitations of this method including simultaneous fault detection, diagnostics of failure cause, and high processing volume are eliminated. The method is implemented on a spacecraft as a case study using the MATLAB/SIMULINK software and demonstrates that the responsive speed and accuracy of the proposed method is significantly much more effective and accurate than the previous method.
\end{abstract}

\section{Keywords:}

Fault detection, Spacecraft, Reliability, Actuator, Model-based, Applicable

\section{Introduction}

Due to the importance, complexity, and cost of the space projects, increasing the reliability of system operation is vitally important. The main purpose is to consider various adverse conditions and takes the required action to attribute these faults or

\footnotetext{
*Corresponding author: aalikhani@ari.ac.ir
} 
failures. Supervisory functions have a significant role to enhance reliability to detect undesired actions and subsequently perform the predesigned operations to maintain system performance.

The supervision function can be classified into three major groups [1]: monitoring, automatic protection action, and the Fault Detection and Diagnosis (FDD) process. In the monitoring, process important variables checked and according to the predefined range, hazardous events are reported to the system management. The first two groups, only able to react after the occurrence a dangerous event or a longlasting steadily increasing fault. The FDD procedure measures variables, states, and considering platform dynamics, detect dangerous or undesired defects.

From the past decades, FDD has been an interesting area of research in aerospace, process controls, automotive, manufacturing, and the nuclear industry to improve systems reliability. Several methods developed in this field, which the substantial difference between them, are the information and knowledge to formulate the system application [2]. Detection can be obtained based on prior knowledge (model-based design according to mathematical equations) or the databased approach using numerous observation and experimental tests (like black-box models, neural networks) [3-5].

The data-driven method does not require mathematical model development but, instead, monitors system behavior by measurement data and actual tests [6-8]. Datadriven methods can be beneficial when the mathematical model of a physical system is complex and is not available. This approach needs a large volume of training data and is limited in extrapolation beyond the training data range. One of the data-driven processes on the vehicle includes current spectrum analysis, vibration, and acoustic analysis for motor fault identification [9-10]. This approach is also used in [11] to detect gearbox faults by using an adaptive method. Capozzoli, Laura, and Khan [12] applied a data-driven method to commercial buildings to detect faults as duct fouling and excessive infiltration. In [13], Khalastchi and Kalech applied sensor-based fault detection and diagnosis (SFDD), which uses the structural model data as well as the correlations between sensor measurements to detect and subsequently diagnose faults in a robot.

There is an increasing interest in the development of model-based FDD methods as can be seen in the many papers submitted to the IFAC (International Federation of Automatic Control) $[14,15]$. The analytical redundancy is often according to these techniques: quantitative model-based methods, parity relations, observers, Kalman- 
filter, and parameter estimations [16-19]. In [20], a Kalman filter-based method was performed for diagnosing both parametric and catastrophic faults in analog circuits. Gliel [21] employed a state observation to detect the fault in an industrial boiler.

In aerospace vehicles, mostly the fault detection and diagnosis process relies on hardware and sensor redundancy [22]. However, adding redundant hardware due to some main reasons as volume, weight, and cost limitations is not possible; supervision system through software has been obtained attraction in recent years. In [23] and [24] cold gas thruster faults study during the Mars express spacecraft using the FDD method. Kobayashi [25] developed a diagnostic system based on a special Kalman filter and evaluate its application to in-flight aircraft engine sensors. Various applications of parameter identification regarding fault diagnosis based on the leastsquares method presented in [26-27].

Another effective approach to detect and isolate actuator or sensor faults is the multiple model adaptive estimation (MMAE) method which was successfully implemented on various vehicles like aircraft and underwater vehicles [28-29]. The main advantage of the MMAE method compare to other FDD algorithms lies in its accuracy, fast responsiveness, and robustness against parameter variations. In addition, the method enables the reconstruction of a correct state estimate even when an actuator or sensor fault occurs. With the combination of the MMAE method with the Extended Kalman Filter called EMMAE, this method is capable to detect the fault in the nonlinear dynamics platform [30-31].

In our previous article [32], the implementation of the EMMAE method on the spacecraft was evaluated and different common faults were applied on the reaction wheel as its actuator. Although the method was effective in detecting different faults and had high speed and proper accuracy, it was not applicable in certain conditions such as simultaneous failures and fault diagnostics due to requiring high processing hardware.

The objective of this paper is to develop the Modified extended multiple model adaptive estimation (MEMMAE) method as a complementary method to solve the EMMAE mentioned problem. In this approach, the recursive least- square (RLS) methods were added to EMMAE methods to enhance the FDD capability to detect faults in all critical conditions using conventional hardware. Different actuator faults are applied and the method performance is evaluated. Furthermore, the ability to determine of dynamics states on a nonlinear system in even fault conditions is discussed. 
The paper is organized as follows: Section 2 deals with the description of the attitude and control subsystem (ADCS) of spacecraft, it's equations and common faults on the spacecraft during its mission. The MEMMAE method, mathematical equations, fault detection performance in various crucial cases using Simulink/Matlab software are provided in section 3. Finally, paper conclusions are presented in section 4.

\section{System Description}

\subsection{Satellite Attitude and Control}

The Attitude and Control Subsystem (ADCS) is one of the main subsystems in the satellite, which is responsible to control the platform to the desired attitude under persistence disturbances and uncertainties. This subsystem consists of different units with specific functions. presence of numerous components as well as complex algorithms has made it the most important and challenging subsystem.

The schematic for a closed-loop process of the ADCS subsystem during satellite mission is presented in Figure 1.

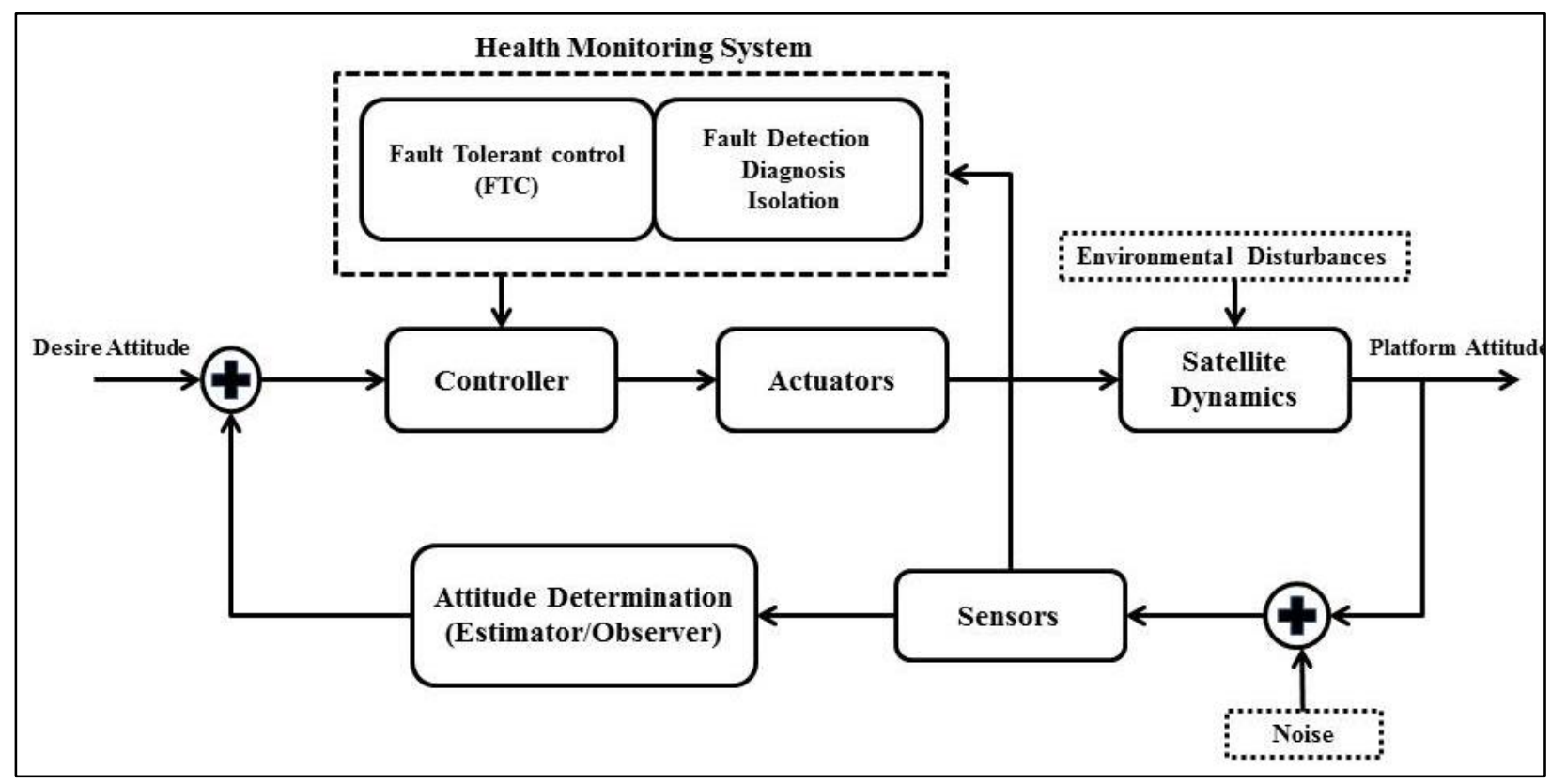

Figure 1. Schematic of a satellite attitude control subsystem [32]

Based on this structure, the controller using sensor data and platform attitude generated the required command and sends it to actuators. By operation of actuators, the platform excited and its attitude changed. Various sensors such as sun sensors, magnetometers, and gyro measure this change and send these measurements to the 
attitude determination unit. During this process, the health-monitoring unit plays a significant role. This unit can detect and diagnose faults. In the case of a fault occurrence, model-based methods can be utilized to detect, diagnose and isolate the problems. In this paper, our focus is on the detection and diagnosis of different faults on the actuators based on model-based algorithms.

\subsection{Typical Actuator-Fault Cases}

By increasing knowledge about satellite components and their behavior in the space environment, usage of the FDD approaches increased markedly. Due to design and mass restrictions, advanced monitoring techniques can compensate the absence of hardware redundancy. Figure 2 shows the failure breakdown for the different spacecraft subsystems.

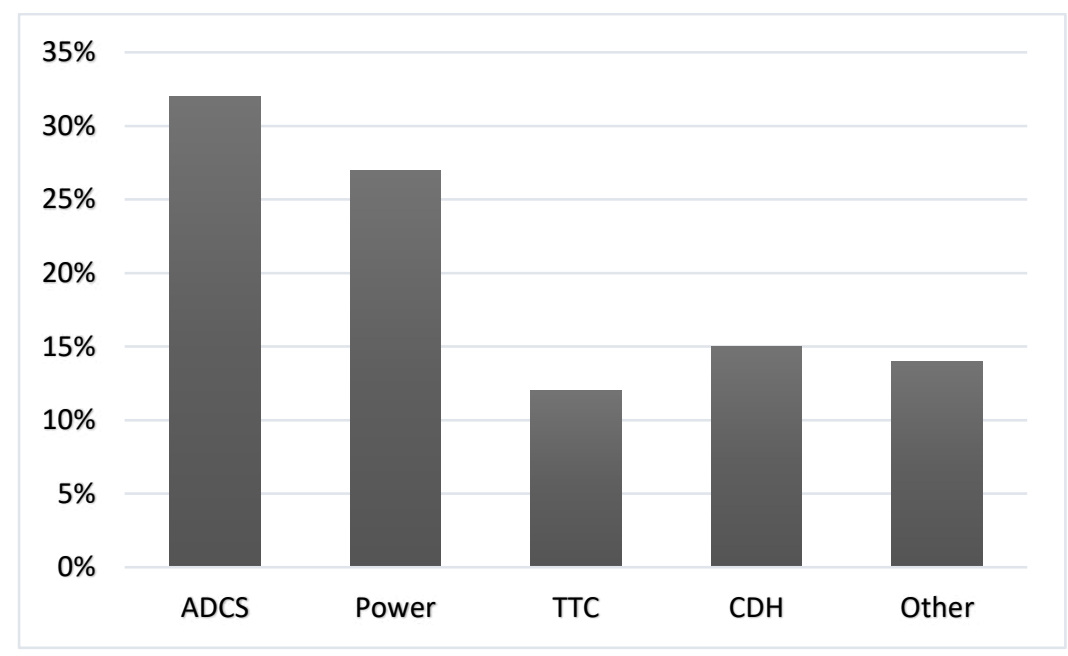

Figure 2. Spacecraft subsystems affected [32]

The most percentage of failure occurrence is related to ADCS in particular its actuators. Actuators consist of moving mechanical parts and are subject to unanticipated faults/failures like cold solder joint, minute particles, or massive temperature fluctuations [33].

Various actuators are employed base on the satellite missions as reaction wheel (RW), Momentum Wheel (MW), cold gas thruster, and magnetic torquer but the most common actuator to provide pointing is RW. A RW consists of a flywheel attached to a brushless DC motor. Notably, that at least three RWs are required for fully actuated attitude control [34].

Table 1 describes some of the recent momentum/reaction wheel failures in space missions concerning the importance of the monitoring system. 
Table1. Summary of on-orbit failure of RWs [35]

\begin{tabular}{ccc}
\hline Spacecraft & Cause of anomaly & Year \\
\hline Radarsat-1 & 2 pitch MWs failed & 1999,2002 \\
ISS & 1 CMG failed on June 8 & 2002 \\
Hayabusa & X and Y axis RW failed on July 31 and October 2, & 2005 \\
& $\begin{array}{c}\text { respectively } \\
\text { FUSE }\end{array}$ & Final RW required for accurate pointing failed \\
TIMED & Single RW failure & 2007 \\
Dawn & Two RW failures due to excessive friction & 2007 \\
& $\begin{array}{c}\text { development } \\
\text { Kepler }\end{array}$ & Two RW failures disabled accurate positioning/data \\
& collection & 2012 \\
& & \\
\hline
\end{tabular}

The main reason for failures in $\mathrm{RW}$ can be classified into four main groups $[36,37]$ : 1. Failure to respond to control signals: due to faulty electronics driver and power supply. The wheel fails to respond to any control signals and decelerates slowly or holds its position. This can cause zero or undesired torque outputs from the wheel.

2. Decreased reaction torque: caused by the friction between rotor and stator, marginal failure of the bearings leads to do not apply desired control commands. 3. Increased bias torque: incipient faults, caused by friction due to aging, or timevarying temperature, etc. can lead to undesired acceleration or deceleration of the wheel. Consequently, the wheel would generate a biasing torque even in the event of zero commanded torque.

4. Continuous generation of reaction torque: intermittent time-varying faults in the motor current or fault in the bus voltage can lead to continuous increase/decrease in the wheel speed. This can lead to undesired reaction torques generated by the wheel regardless of the commanded torque by the controller.

\subsection{Mathematical Models}

Euler equations are used to model the dynamics of the spacecraft, with respect to an inertial coordinate system $\{\mathrm{I}\}$. The reaction wheels are used as excitation actuators. The attitude dynamics of a rigid simulator are given by Euler's equation [34, 38]: 


$$
\vec{M}_{e x t}=J \overrightarrow{\dot{\omega}}+\vec{\omega} \times J \vec{\omega}-\vec{T}_{r w}-\vec{\omega} \times \vec{h}_{r w}
$$

where $J$ is the spacecraft's inertia matrix, $\omega=\left[\begin{array}{lll}\omega_{x} & \omega_{y} & \omega_{z}\end{array}\right]^{T}$ is the angular velocity of $\{\mathrm{B}\}$ in $\{\mathrm{I}\}, \vec{h}_{r w}$ is the angular momentum of the reaction wheels and $\vec{M}_{\text {ext }}$ is the external torque which is applied on the platform. In addition, this external torque contains various types as:

$$
\vec{M}_{\text {ext }}=\vec{M}_{\text {aero }}+\vec{M}_{\text {sun }}+\vec{M}_{g}+\vec{M}_{m}
$$

Where $\vec{M}_{\text {aero }}$ is the aerodynamics torque, $\vec{M}_{\text {sun }}$ is applied from solar pressure, $\vec{M}_{g}$ is the major torque and from gravity gradient and $\vec{M}_{m}$ created from interaction of satellite with the magnetic field. The rotational kinematics using a quaternion can be described by

$$
\left[\begin{array}{l}
\dot{q}_{1} \\
\dot{q}_{2} \\
\dot{q}_{3} \\
\dot{q}_{4}
\end{array}\right]=\frac{1}{2}\left[\begin{array}{cccc}
0 & \omega_{3} & -\omega_{y} & \omega_{x} \\
-\omega_{z} & 0 & \omega_{x} & \omega_{y} \\
\omega_{y} & -\omega_{x} & 0 & \omega_{z} \\
-\omega_{x} & -\omega_{y} & -\omega_{z} & 0
\end{array}\right]
$$

where $\mathrm{q}=\left[\begin{array}{llll}\mathrm{q}_{1} & \mathrm{q}_{2} & \mathrm{q}_{3} & \mathrm{q}_{4}\end{array}\right]^{\mathrm{T}}$ are the quaternions. The quaternion is defined as a vector in the following way

$$
q=i q_{1}+j q_{2}+k q_{3}+q_{4}
$$

The mathematical model of a reaction wheel is similar to the model of a DC motor, in which the flywheel adds to increase the total inertia and provide much more torque. In the friction model considered here, the viscous friction, Coulomb friction, and the friction of Stribeck are included. The equation describing the friction torque is as [39]: 


$$
T_{r w}=J \dot{w}+b w+\operatorname{sgn}(w)\left[c+d e^{-\frac{w^{2}}{w_{s}^{2}}}\right]
$$

where $T_{r w}$ is the motor torque, the wheel's and rotor inertia is $J, b$ is the coefficient of viscous friction, $c$ is the Coulomb friction torque, $d$ is the starting torque, $w$ is the angular velocity of the wheel, and $w_{S}$ is known as Stribeck speed.

\section{Modified EMMAE Method}

The developed method is according to completing the EMMAE method and presents an innovative technique to solve its limitations. Despite the many advantages of the method, due to the structure and equations of this method, some limitations were also observed in software implementation.

1- The method cannot detect faults simultaneously. According to the probablity Equation, which assigns a probability to different states of failure, the probability is divided between different states. In other words, if faults occur in series, they can detect, but in the event of parallel and simultaneous faults, only one actuator is identified as faulty.

2- Regarding the ability of diagnostics, although it is possible in programming and software, due to the many causes of failure and a large number of actuators in different systems such as spacecraft, a very high processing volume is required. In the diagnostics process, a large number of EKFs are executed in parallel, which in hardware implementation cannot perform practically.

3- The sensitivity of the method is the responsibility of the programmer. The system can detect the error with the least deviation from the desired value and can remain reluctant with a high difference. Therefore, it requires a high level of experience in hardware implementation.

The main approach of this method is to create the ability to implement practically on various dynamic systems, including spacecraft. The process of implementing the MEMMAE method is explained in Fig.3. 


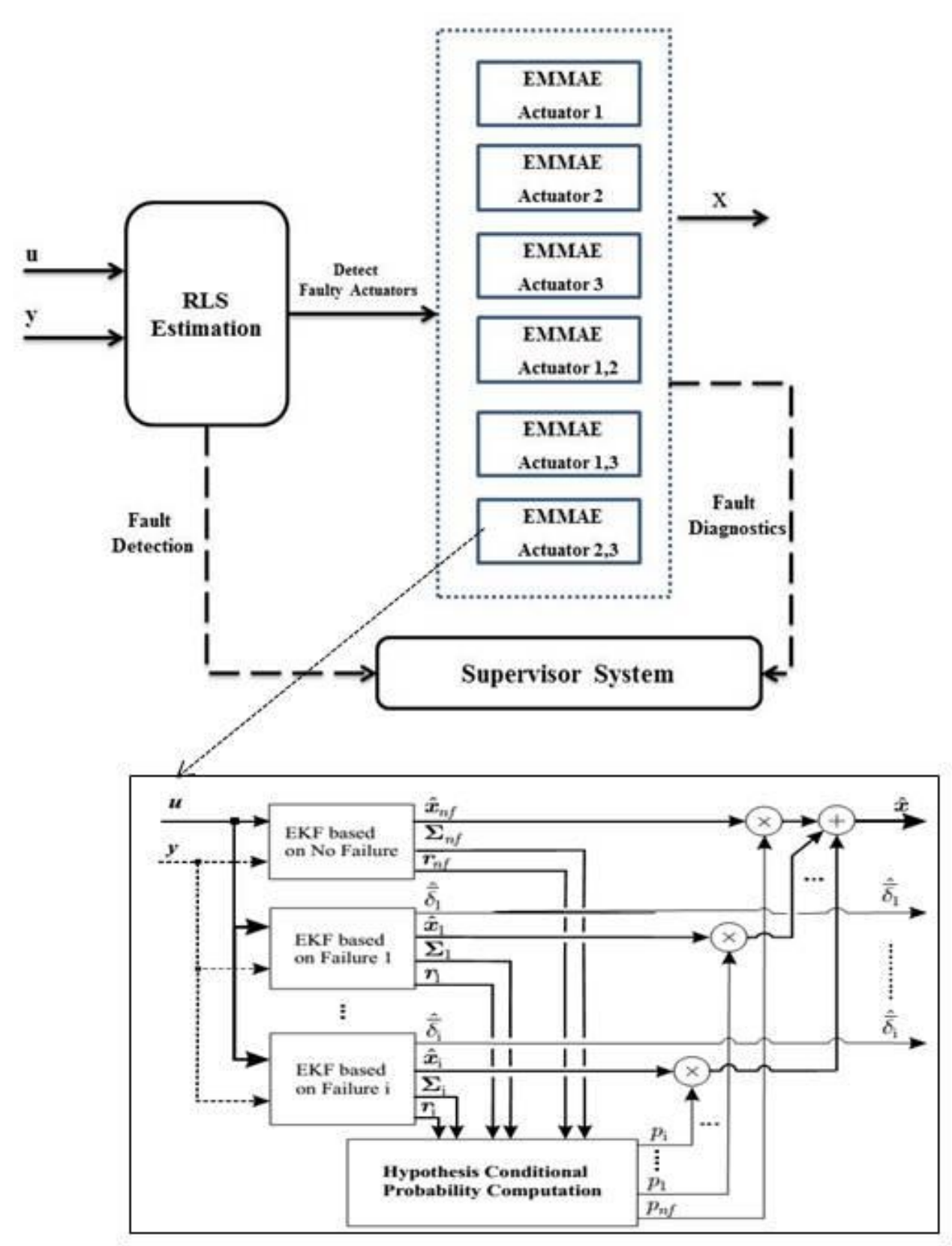

Figure 3. The MEMMAE method Scenario

This technique consists of two main parts: fault detection and fault diagnostics. In the detection part, using the Recursive Least-square (RLS) method, faulty actuators are identified. In addition to high accuracy and responsive speed, it can detect faults simultaneously. 
In the second part, the EMMAE method is used and the cause of the fault is determined. Each faulty actuator may be faced with a defect for a variety of reasons, which are recognized in this section. A set of EKFs corresponding to different causes runs parallel, and the failure cause is determined based on the probability method.

The valuable point in this scenario is the identification of the faulty actuators at the first step. This initial identification causes that only the subsystem concerning actuators become activate, resulting in a significant decrease in the number of EKFs. Other advantages of this method are robustness against uncertainties and disturbances of the environment. defining the level of sensitivity based on practical and software implementations can provide good reliability in addition, adding RLS technique leads to improve performance and make the ability to run this approach on conventional hardware

\subsection{Fault Detection (Step 1)}

The Recursive Least-Square (RLS) is one of the most well-known algorithms used in adaptive filtering, system identification, and adaptive control. Its popularity is mainly due to its fast convergence speed, which is considered optimal in practice. In this method, the estimated parameters are computed recursively over time: By converting problem to the regression form such that $\mathrm{Ax}=\mathrm{B}$, suppose we have an estimated $x_{t-1}$ at iteration $\mathrm{t}-1$, then recursive identification aims to compute a new estimated $x_{t}$ by a simple modification of $x_{t-1}$ when a new observation becomes available at iteration $t[40,41]$.

The RLS approach starts from a slightly modified loss function:

$$
V(t)=\sum_{s=1}^{t} \lambda^{t-s}\left(B_{t}-A_{t}{ }^{T} x\right)
$$

Where $A$ and $B$ are known matrix from estimation problem and $\lambda$ is forgetting factor. The main approach of the algorithm is find $\mathrm{x}$ to reduce loss function.

The RLS algorithm can be represented by Eq.(7) as follows[42]:

$$
\begin{array}{r}
K(t)=\frac{\lambda^{-1} P(t-1) A(t)}{1+\lambda^{-1} A^{T}(t) P(t-1) A(t)} \\
\propto(t)=B(t)-A(t)^{T} x(t-1)
\end{array}
$$




$$
\begin{aligned}
& x(t)=x(t-1)+K(t) \propto(t) \\
& P(t)=\lambda^{-1} P(t-1)-\lambda^{-1} K(t) A(t)^{T} P(t-1)
\end{aligned}
$$

Where $\propto(t)$ is an estimation error at the moment $t$ and $\lambda$ is forgetting factor, If $\lambda$ is set to values slightly smaller than 1 ( $\lambda=0.99$ or $\lambda=0.95)$, for increasing $t$ past observations are discounted. The assumed $\lambda$ becomes smaller, the quicker information obtained from previous data will be forgotten. Being initialized with $P(0)=\delta^{-1} I$, where $\delta$ is a small positive constant. The advantage of the RLS algorithm is that there is no need to invert matrices, in addition to its saving computational power.

\subsection{Step 2: Fault Diagnostics}

One effective scenario for diagnostics faults on actuators or sensors is the EMMAE method as shown in Fig3. It monitors the system based on a bank of Extended Kalman Filters (EKFs) running in parallel, which each of these EKFs correspondings to the specific status of the system.

A hypothesis algorithm by comparing the residuals of each EKFs estimation and system outputs provides a conditional probability to each fault hypothesis.

The main advantage of the EMMAE method attributes the responsiveness to parameter variations, leading to fault isolation more quickly comparing other methods. The scenario also reconstructs and prepares the correct state estimation under the occurrence of actuator or sensor fault. This capability is based on the summing of each EKF estimated and weighting them using its corresponding probability.

\subsubsection{EKF design process}

The EKFs are designed based on a set of continuous nonlinear differential equations. The prediction phase of the estimation can be determined as [43]:

$$
\begin{gathered}
\hat{x}_{k \mid k-1}=F_{k} \hat{x}_{k-1 \mid k-1}+G_{k} u_{k} \\
P_{k \mid k-1}=F_{k} P_{k-1 \mid k-1} F_{k}^{T}+Q_{k}
\end{gathered}
$$


Where $F_{k}$ is the continuous system dynamics matrix, $Q$ the covariance of the process noise is, $G$ is the control-input model and $P$ is the covariance matrix. In the following, the update phase is presented [44-45].

$$
\begin{gathered}
\hat{y}_{k}=z_{k}-H_{k} \hat{x}_{k \mid k-1} \\
S_{k}=H_{k} P_{k \mid k-1} H^{T}{ }_{k}+R_{k} \\
L_{k}=P_{k \mid k-1} H^{T}{ }_{k} S_{k}{ }^{-1} \\
\hat{x}_{k \mid k}=\hat{x}_{k \mid k-1}+L_{k} y_{k} \\
P_{k \mid k}=\left(I-L_{k} H_{k}\right) P_{k \mid k-1}
\end{gathered}
$$

Where $R$ is the covariance of the observation noise and the $H$ is the observation model.

\subsubsection{EKF reproduction based on the faulty actuator}

The state vector of the $i^{\text {th }}$ is augmented to monitor the actuator faults. This stated is added to the estimate during EKF estimation. Therefore, the state vector for each filter $i$ is [43]:

$$
z_{i}=\left[\begin{array}{l}
x \\
\delta_{i}
\end{array}\right]
$$

The augmented state vector leads to the following state space equations.

$$
\begin{gathered}
z_{i}(k+1)=f_{z i}\left(z_{i}(k), \delta(k)\right)+w(k) \\
y_{i}(k)=h\left(z_{i}(k)\right)+v(k)
\end{gathered}
$$

where,

$$
f_{z i}\left(z_{i}(k), \delta(k)\right)=\left[\begin{array}{c}
f_{z i}\left(z_{i}(k), \delta(k)\right) \\
\delta_{i}(k)
\end{array}\right]
$$

The linearization of the dynamics matrix yields 


$$
F_{z i}(k)=\left.\frac{\partial}{\partial_{z i}} f_{z i}\left(z_{i}(k), \delta(k)\right)\right|_{z_{i}=\widehat{z}_{l}(k \mid k)}=\left[\begin{array}{cc}
F(k) & G^{(i)}(K) \\
0 & 1
\end{array}\right]
$$

Where $G^{(i)}$ represents the $i^{\text {th }}$ column of $G$. The input matrix will be:

$$
G_{z i}(k)=\left.\frac{\partial}{\partial_{\bar{\delta} i}} f_{z i}\left(z_{i}(k), \delta(k)\right)\right|_{z_{i}=\widehat{z}_{l}(k \mid k)}=\left[\begin{array}{c}
G^{(0, i)}(K) \\
0
\end{array}\right]
$$

With $G^{(0, i)}$ representing the matrix $G$ with its $i^{\text {th }}$ column set to zero. The linearization of the measurement matrix is

$$
H_{z i}(k)=\left.\frac{\partial}{\partial_{z i}} h\left(z_{i}(k), \delta(k)\right)\right|_{z_{i}=\widehat{z}_{l}(k \mid k)}=\left[C_{x}(k) \quad C_{\bar{\delta}}(k)\right]
$$

with

$$
\begin{gathered}
C_{x}(k)=\left.\frac{\partial}{\partial_{x}} h\left(z_{i}(k)\right)\right|_{z_{i}=\widehat{z}_{l}(k \mid k)}=H \\
C_{\bar{\delta} i}(k)=\left.\frac{\partial}{\partial_{\bar{\delta} i}} h\left(z_{i}(k)\right)\right|_{z_{i}=\widehat{z}_{l}(k \mid k)}=0
\end{gathered}
$$

Using the above equations, the linearized system evaluated at each sampling time can be shown as:

$$
\begin{gathered}
{\left[\begin{array}{c}
x(k+1) \\
\bar{\delta}(k+1)
\end{array}\right]=\left[\begin{array}{cc}
F(k) & G^{(i)}(K) \\
0 & 1
\end{array}\right]\left[\begin{array}{l}
x \\
\bar{\delta}_{i}
\end{array}\right]+\left[\begin{array}{c}
G^{(0, i)}(K) \\
0
\end{array}\right] \delta(k)} \\
y k)=\left[\begin{array}{ll}
H & 0
\end{array}\right]\left[\begin{array}{l}
\frac{x}{\delta_{i}}
\end{array}\right]
\end{gathered}
$$

\subsection{3 hypothesis testing algorithm}

A hypothesis-testing algorithm uses the residuals and the state error covariance matrix from each EKF to assign a conditional probability to each fault scenario. The 
estimated state vector of the system is the sum of the state vector of each EKF weighted by its corresponding probability [43],

$$
\hat{x}[k]=\sum_{i} \hat{x}_{i}[k] \cdot p_{i}[k]
$$

where $\hat{x}_{i}[k]$ the state estimate is computed by the EKF that assumes the fault scenario $\theta_{i}$. The index icovers all the fault scenarios implemented, including the nofault case. The fault probability $p_{i}[k]$ can be expressed as the posteriori conditional probability $p_{i}[k]=p\left(\theta=\theta_{i} \mid Y_{k}\right)$ the probability that the actual plant can be categorized in scenario $\theta_{i}$ given the sequence of the last measurements $Y_{k}$.

By examining the probabilities computed by $\mathrm{Eq}(19)$, we can determine the health status of the system, either in the no-fault case or in an actuator/sensor failure case[43,31]:

$$
p_{i}[k]=p\left[\theta=\theta_{i} \mid Y_{k}\right]=\frac{p\left[y=y_{k} \mid\left(\theta=\theta_{i}, Y_{k-1}\right)\right] \cdot p_{i}[k-1]}{\sum_{j=0}^{N} p\left[y=y_{k} \mid\left(\theta=\theta_{j}, Y_{k-1}\right)\right] \cdot p_{j}[k-1]}
$$

Since a fault may occur at any time, regardless of which actuator may fail, we decide to assign the same probability to all the scenarios, i.e., $p\left[\theta=\theta_{i}\right]=1 / N$ for $\mathrm{j}=1, \mathrm{~N}$.

We now derive an explicit formula for the term $p\left[y=y_{k} \mid\left(\theta=\theta_{i}, Y_{k-1}\right)\right]$, which corresponds to the probability of obtaining the measurement data $y_{k}$ at timet $t_{k}=$ $K T_{s}$. The probability density is chosen to be a Gaussian function with its characteristic bell-shaped curve according to the following formula [44-46]:

$$
p\left[y=y_{k} \mid\left(\theta=\theta_{i}, Y_{k-1}\right)\right]=\epsilon_{i}[k] e^{-r_{i}[k]^{T} \sum_{i}^{-1}[k] r_{i}[k] / 2}
$$

Where

$$
\epsilon_{i}[k]=\frac{1}{2 \pi^{m / 2}\left|\sum_{i}(k)\right|^{1 / 2}}
$$

Where term $|\ldots|$ denotes the determinant of the matrix, $m$ represents the measurement dimension, and $\sum_{i}(k)$ is the residual covariance matrix calculated at a 
time step by the $i^{\text {th }}$ EKF. The term $r_{i}[k]$ corresponds to the residuals of the $i^{\text {th }}$ EKF when the measurement update step occurs according to the equation.

$$
r_{i}[k]=y_{k}-h\left(\widehat{x}_{l}(k \mid k-1)\right)
$$

\subsection{Simulation Results of MEMMAE Method}

In order to evaluate the performance of the developed method, this technique is applied on a satellite. Mostly, the satellite uses 3 reaction wheels to control its attitude. The purpose of this method is to detect and diagnostics various faults during the satellite mission.

Six different failure modes can cover all failure states. These fault modes can be described as:

Table2. The main features of defined maneuver (Detection test)

\begin{tabular}{|c|c|}
\hline Fault Mode & Explanation \\
\hline 1 & RW1 \\
\hline 2 & RW2 \\
\hline 3 & RW3 \\
\hline 4 & RW1-RW2 \\
\hline 5 & RW1-RW3 \\
\hline 6 & RW2-RW3 \\
\hline
\end{tabular}

Also, the most common reason for faults on the RW is related to the viscous changes as well as the performance characteristics of the DC motor. The developed algorithm should be able to estimate the cause of the fault, which is mainly due to these two parameters.

First, a slew maneuver without any fault occurrence is performed to ensure about simulation process and designed PID controller on the platform. The initial condition $[0,0,0]$ is considered and the platform is excited to achieve the desire attitude degree that is $[60,40,80]$. 


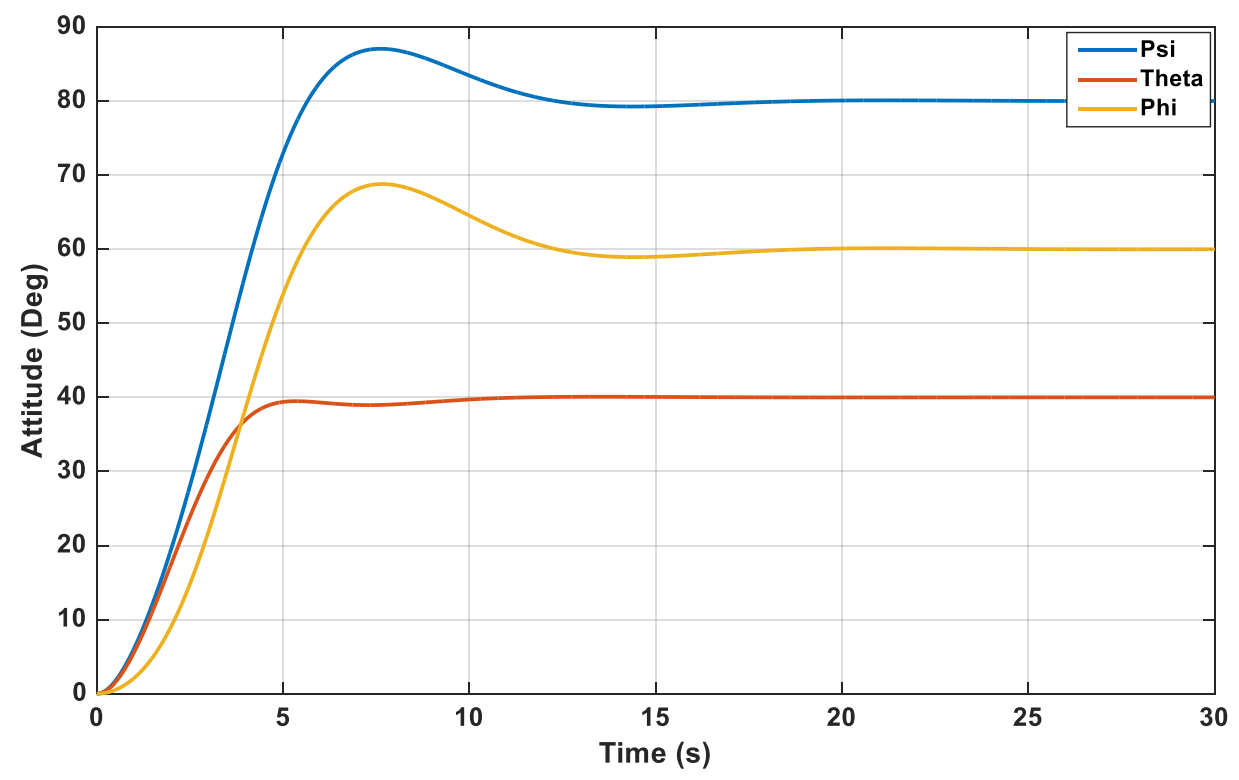

Figure 4. Satellite attitude maneuvered

As can be observed a 3-axis maneuver was implemented on the platform reaching the desired attitude below $30 \mathrm{sec}$ using three reaction wheels as actuators. Also, the satellite angular velocities on the three axes are presented.

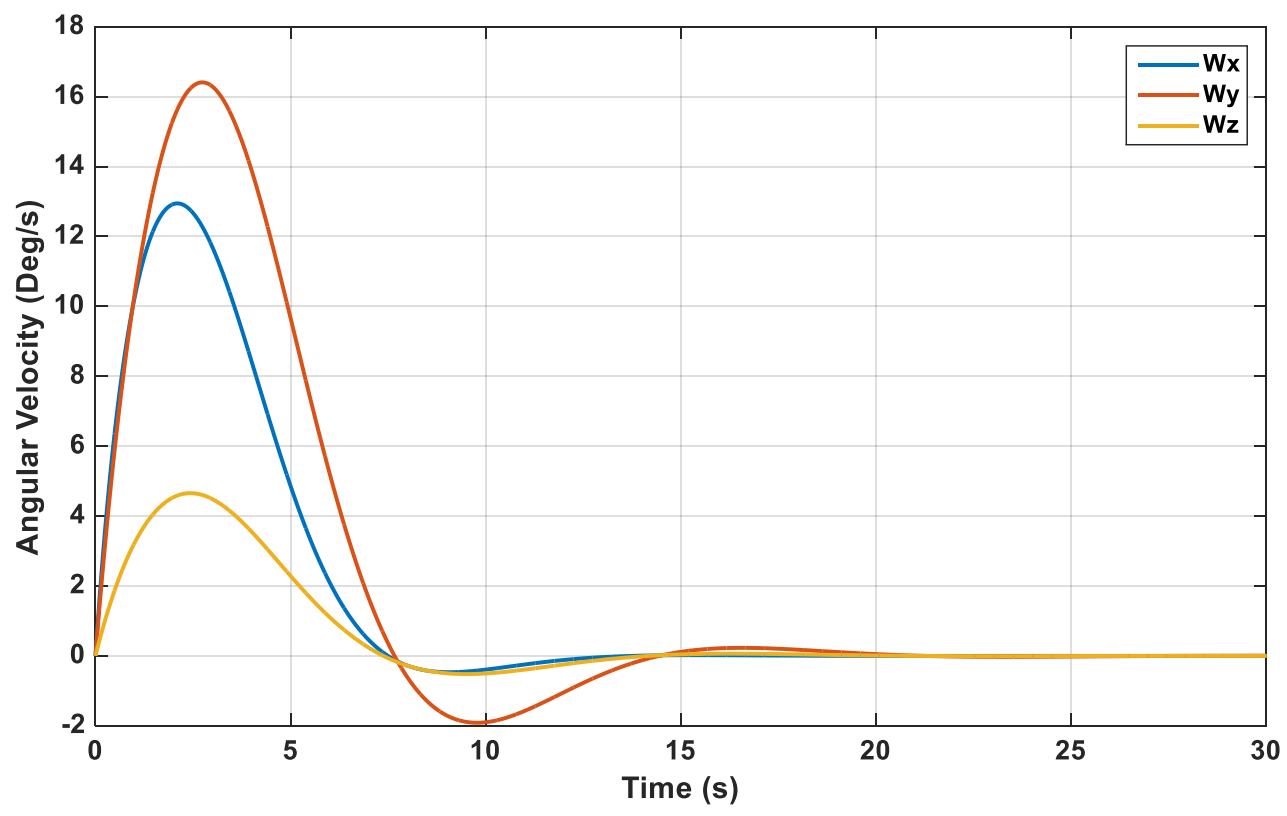

Figure 5. Satellite angular velocities 
The angular velocity increased during the applied maneuver and provides sufficient stability at the end of the maneuver by reaching the desired attitude. The change of actuator velocity is also illustrated to describe this maneuver in detail.

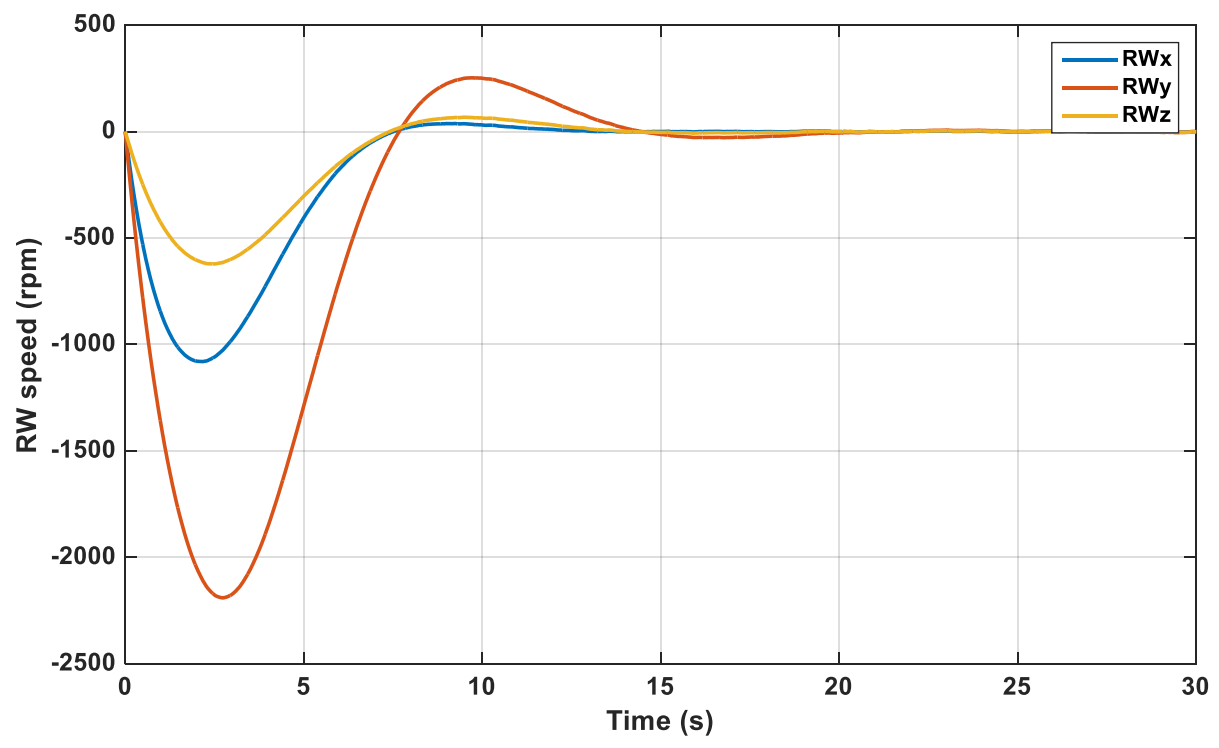

Figure 6. Reaction wheel angular velocity

It is evident that platform momentum remains constant during the mission; hence the satellite angular velocity and actuators are applied in the opposite direction. The maximum actuator velocity is under $2500 \mathrm{rpm}$ and the difference between axes measurements are based on the platform inertia. After ensuring about developed attitude controller performance and mission simulation without occurrence fault, we start to apply different types of actuator faults and assessment the MEMMAE performance to detect the faulty actuator.

\section{Case 1: Fault detection process}

As indicated, the proposed method consists of two parts that run subsequently: fault detection and diagnostics. In the first step, which is based on the RLS algorithm, the defect or fault occurrence in the actuators is estimated.

To investigate the algorithm performance, a sinusoidal maneuver is designed and operators subsequently encountered different faults. The main features of this maneuver are defined as: 
Table3. The main features of defined maneuver (Detection test)

\begin{tabular}{|c|c|c|}
\hline Fault Mode & Faulty actuators & Time \\
\hline $\mathbf{0}$ & N0-Fault & $0-15$ \\
\hline $\mathbf{1}$ & RW1 & $15-30$ \\
\hline $\mathbf{0}$ & N0-Fault & $30-45$ \\
\hline $\mathbf{4}$ & RW1-RW2 & $45-60$ \\
\hline $\mathbf{0}$ & N0-Fault & $60-75$ \\
\hline $\mathbf{2}$ & RW2 & $75-90$ \\
\hline $\mathbf{0}$ & N0-Fault & $90-105$ \\
\hline $\mathbf{6}$ & RW2-RW3 & $105-120$ \\
\hline $\mathbf{0}$ & N0-Fault & $120-135$ \\
\hline $\mathbf{0}$ & N0-Fault & $135-150$ \\
\hline
\end{tabular}

As presented in Table3, various faults are applied to the actuators during the maneuver. to study the ability of the developed method to detect simultaneous faults, these types of failures also occurred in this test. The changes of RW speeds during this test are presented in Fig.7.

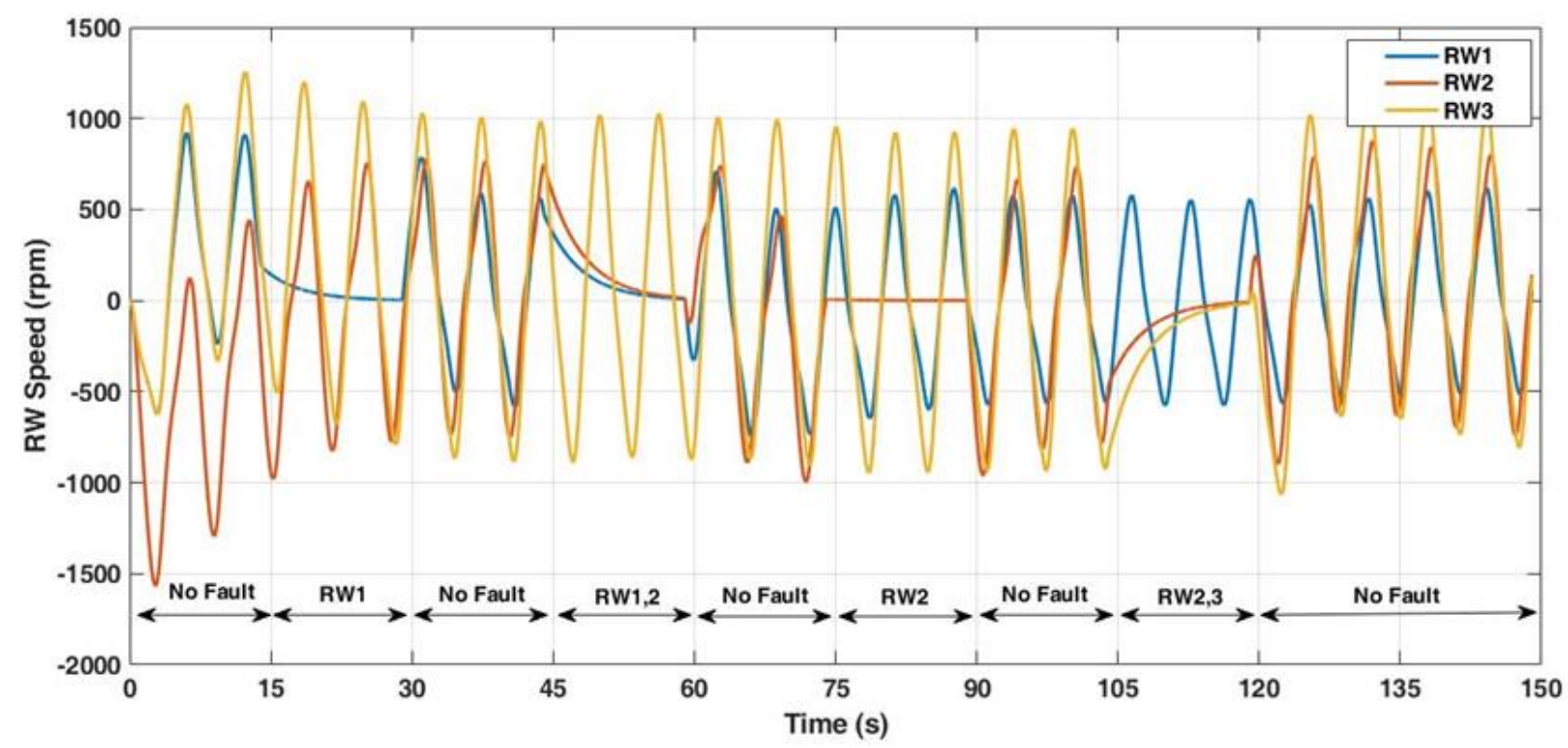

Figure 7. Reaction wheel speed during the fault detection test

As can be seen, due to sinusoidal maneuvering, the wheel changes are sinusoidal. In this test, the error is assumed a complete failure in which no electric current is applied to the RWs. Due to frictional torque, the speed of the motors decreases after 
the cutting of the currents, and this is evident in the changes of speeds. The estimated results of the method are provided in fig. 8

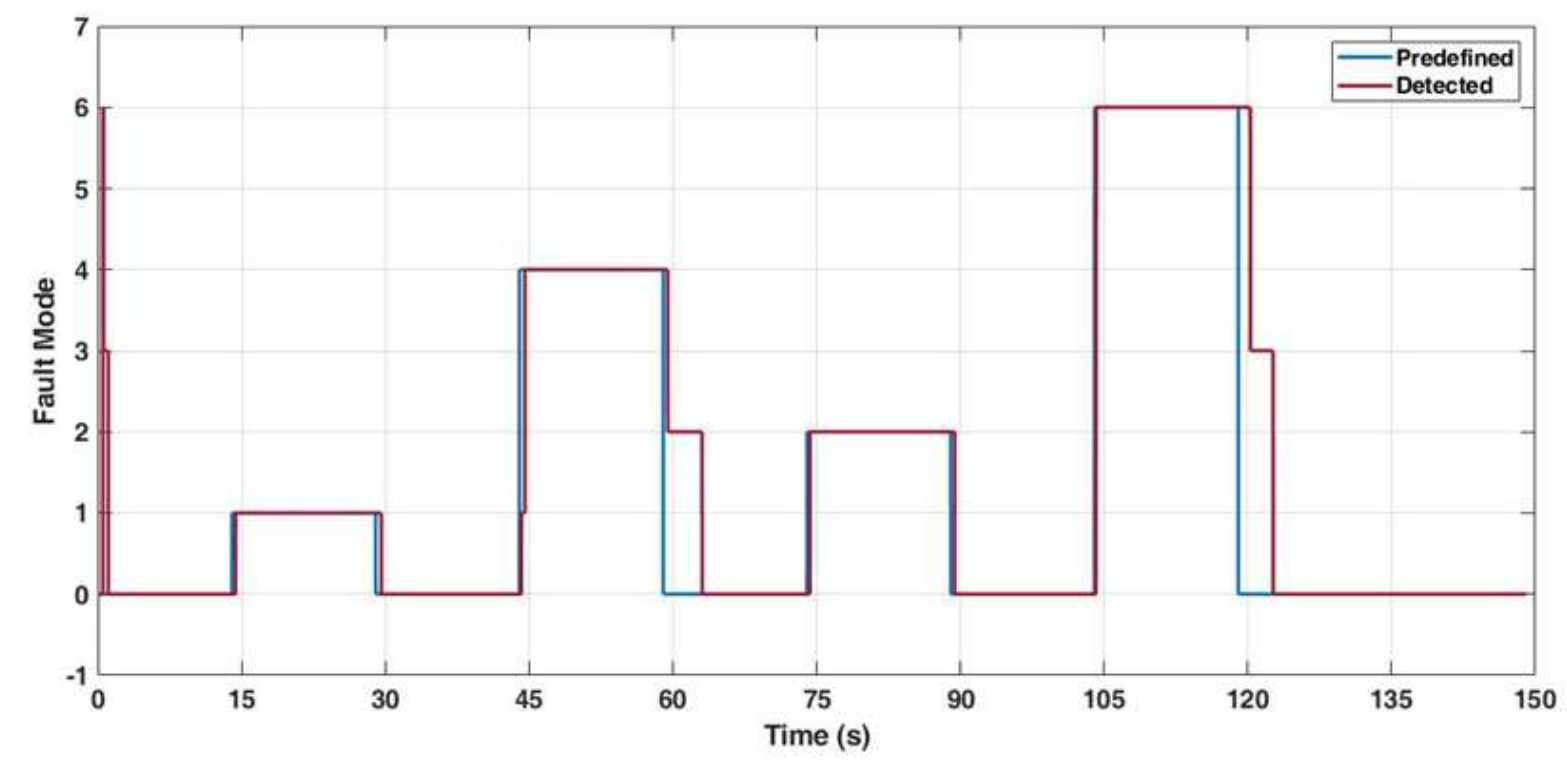

Figure 8. The fault detection results of the AEMME method

the developed method can identify the occurred faults accurately. The algorithm and the predefined (blue line) recognize all fault modes and detected (red line) results follow each other. The maximum time to detect a fault is about 3 seconds, which should be considered. After this period, the monitoring system must make the required decision. Modes 4 and 6 are related to simultaneous failure, which the algorithm has been able to accurately detect.

\section{Case 2: Fault diagnostics process}

After ensuring the performance of the fault detection step in the previous test, the fault diagnostics and the causes of the defects are examined in this test. In this test, a pointing maneuver in which the physical characteristics of a RW change, are simulated. The purpose of this test is to detect the faulty RW and in the second step determination of the cause of this failure. The predefined features of this test are as Table4. 
Table4. The main features of defined maneuver (Diagnostics estimation)

\begin{tabular}{|c|c|}
\hline Title & Explanation \\
\hline Desire Attitude & {$[40-60$ 80] deg } \\
\hline Faulty RW & RW1 \\
\hline Faulty Mode & 1 \\
\hline Cause of defect & Viscose parameter(b) increase from 4e-06 to 4e-04 \\
\hline
\end{tabular}

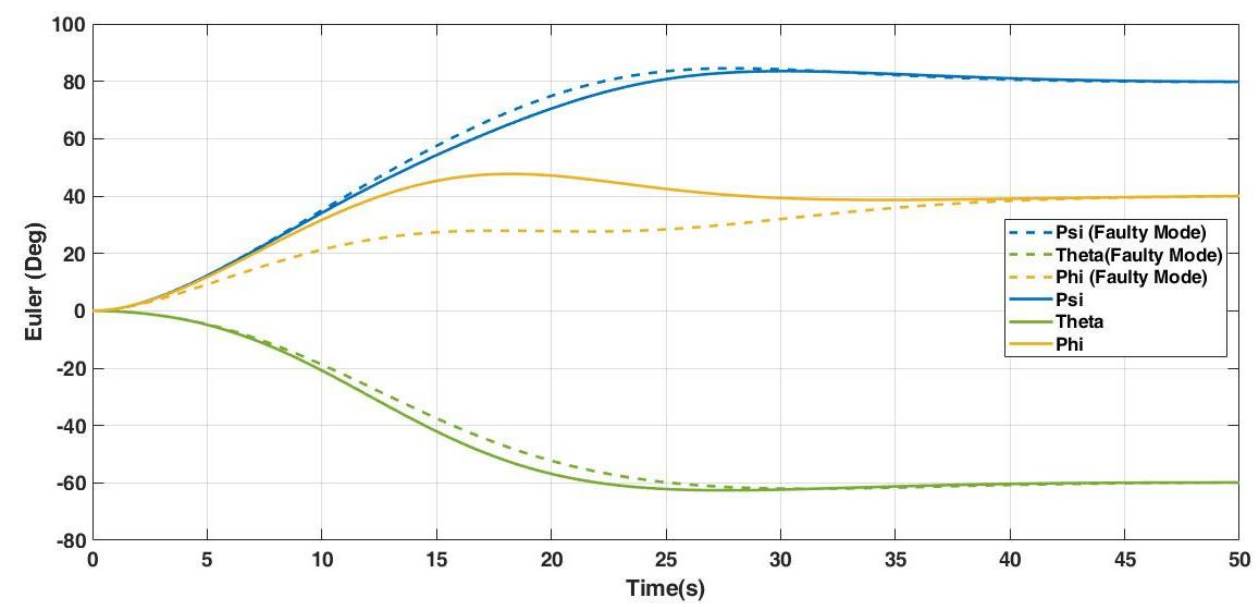

Figure 9. The Euler angles during the diagnostic detection test

As can be seen, both maneuvers can achieve the desired points. Due to the higher frictional torque, the arrival time in the faulty test is longer. This interception delay is more distinguished in wheel 1.

The change of RW speed during this test is reported in Fig.10

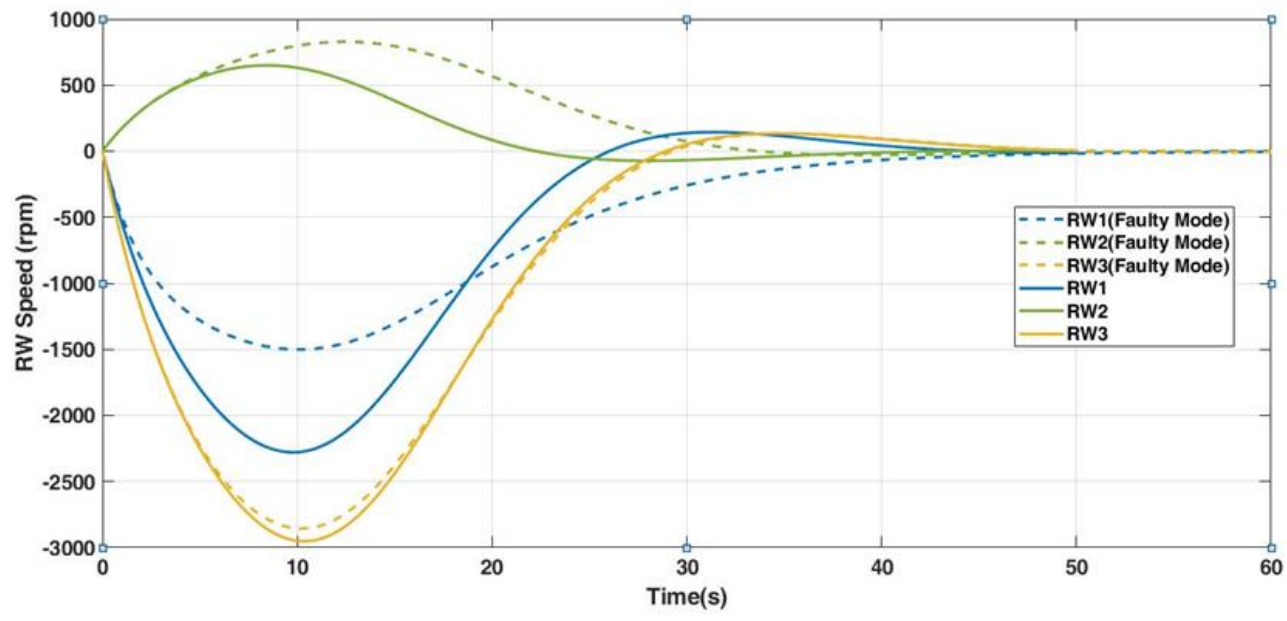


Figure 10. Reaction wheels speed during the fault diagnostics test

The presence of frictional torque reduces the required torque and has a direct effect on RW speed. Due to the coupling dynamics of the spacecraft, this lack of torque supply also affects the motion of other wheels. The difference between the speed of RW1, in the healthy and defective condition, is significant.

The result of the MEMMAE method during this test is provided as Fig.11

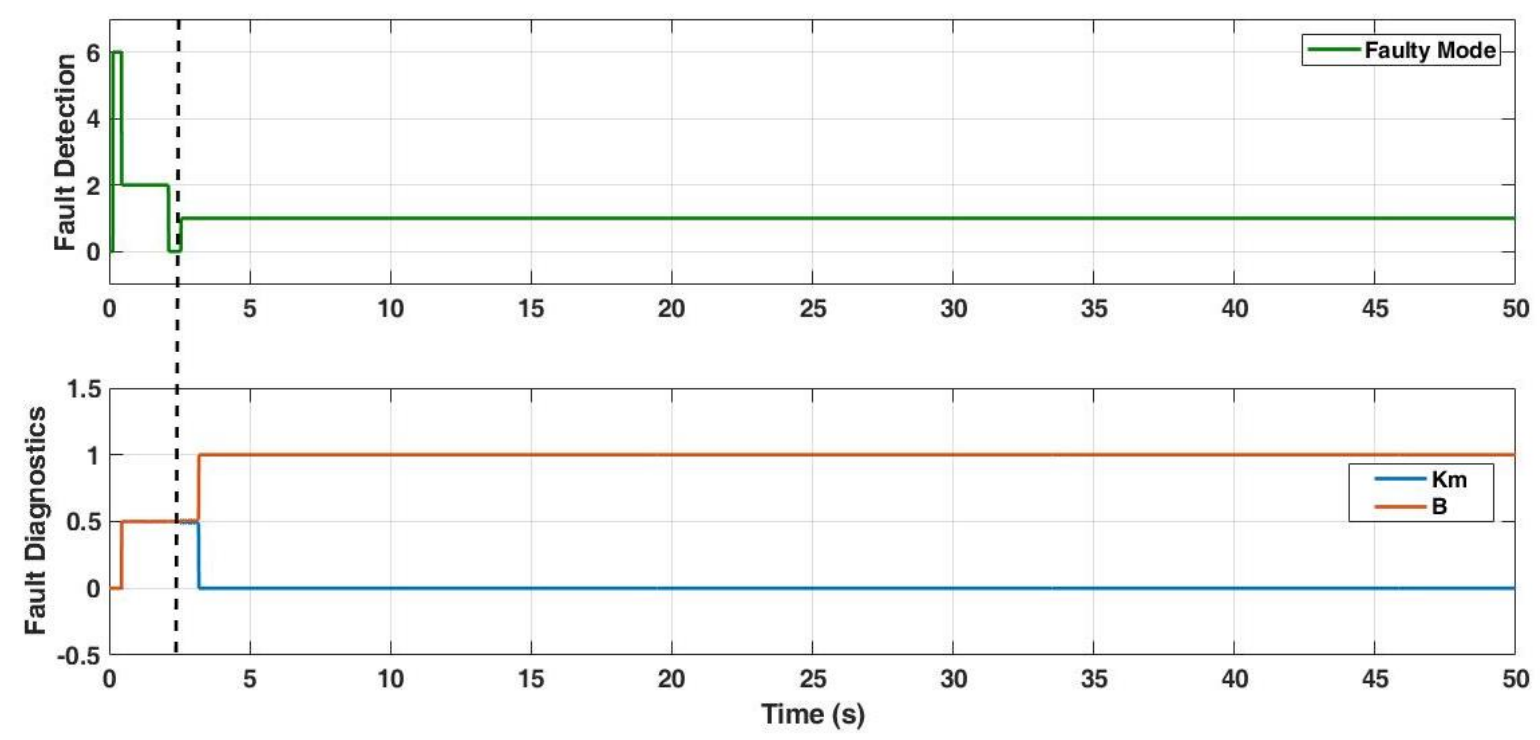

Figure 11. The fault detection and diagnostics results of the MEMME method The algorithm was able to detect the faulty RW in 2 seconds. During this time, the second part of the algorithm is inactive. As can be seen, the probability of defects in both possible cases is $50 \%$.

After two seconds and an accurate estimation of the faulty actuator, the second part is activated and can determine the cause of the fault. Change the Viscose parameter is the cause of the specified failure.

\section{Case3: Compare results with EMMAE}

To compare the efficiency and performance of the developed with the previous method, a scenario has been designed to show the capabilities and improvements of the proposed method. 
The main features of this test are defined as:

Table5. The main features of defined maneuver (Evaluation test)

\begin{tabular}{|c|c|c|}
\hline Fault Mode & Faulty actuators & Time \\
\hline $\mathbf{0}$ & N0-Fault & $0-14$ \\
\hline $\mathbf{1}$ & RW1 & $14-28$ \\
\hline $\mathbf{0}$ & N0-Fault & $28-44$ \\
\hline $\mathbf{6}$ & RW1-RW2 & $44-55$ \\
\hline
\end{tabular}

As can be seen, both simultaneous failure and single failure are considered for evaluation. The changes of RW speeds during this test are presented in Fig.12.

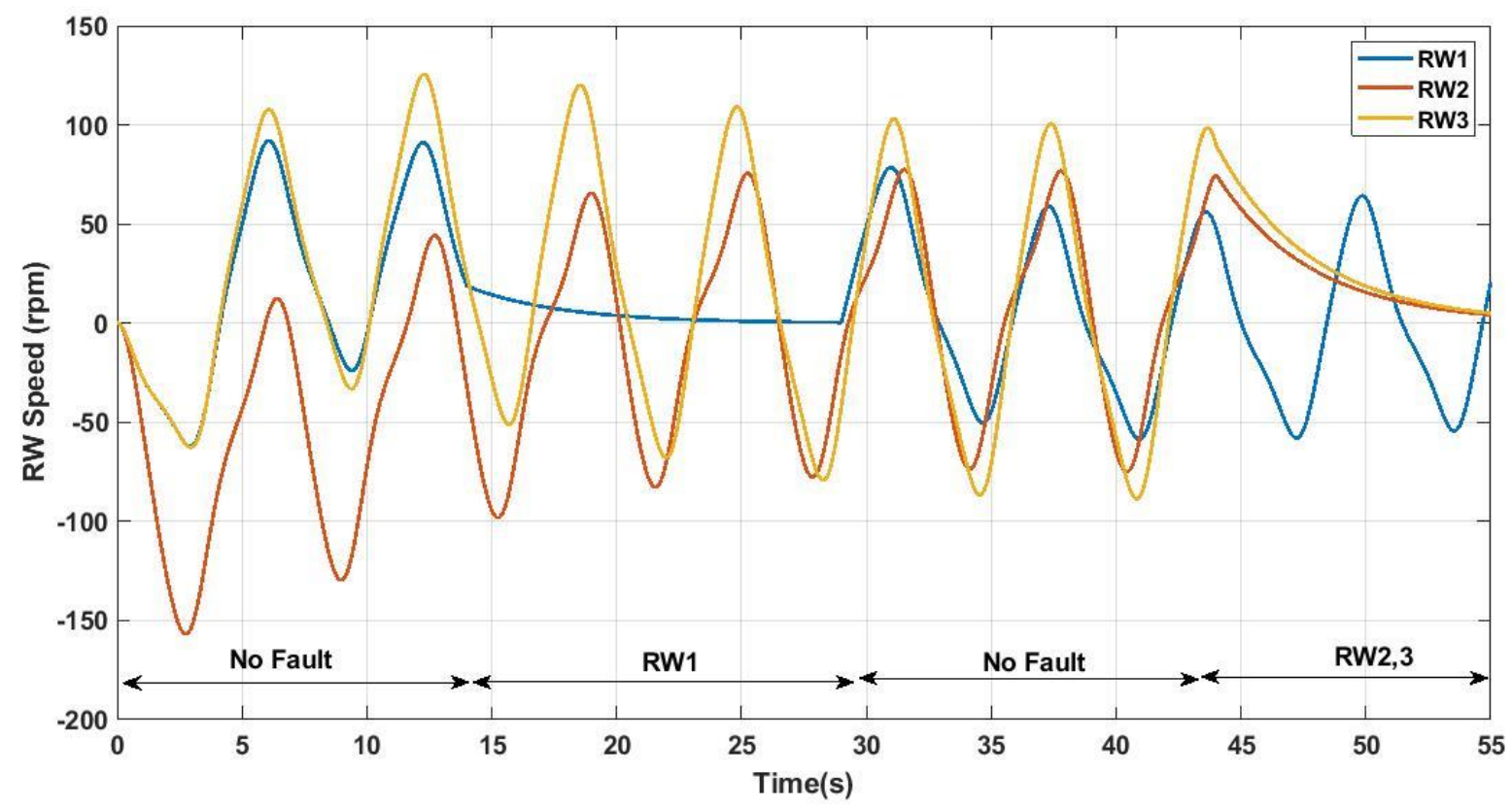

Figure 12. Reaction wheel speed during the evaluation test

To compare the results of the two methods, sinusoidal maneuvers are applied. After deactivating the wheels, their speed decreases due to friction.

The results of the two methods can be observed in Fig.13. 


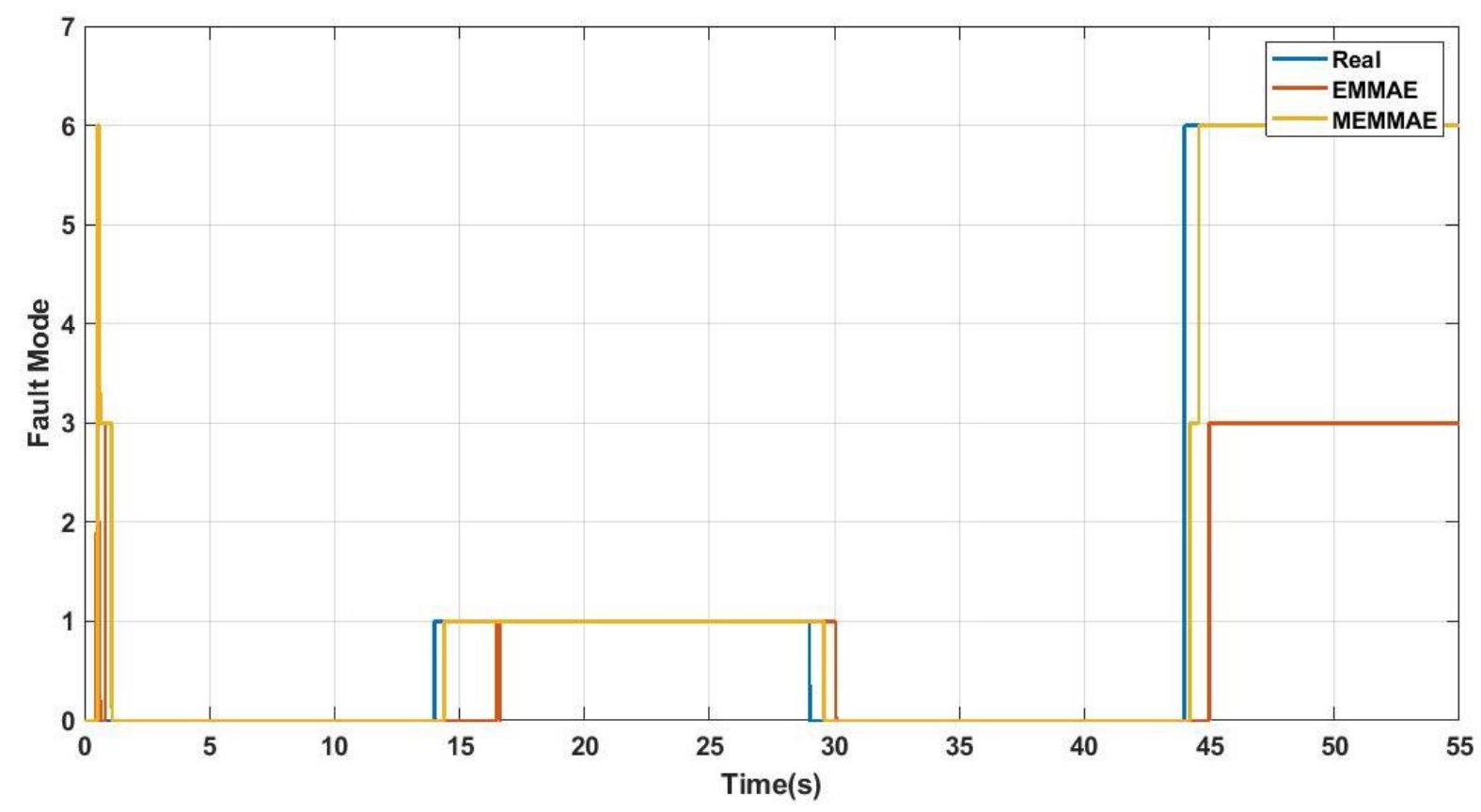

Figure 13. The fault detection results of the EMMAE and MEMMAE method Due to the inability of the EMMAE method to fault diagnostics terms, only the fault detection mode is compared with each other. Both methods correctly identify the first applied faults during 14-278 s. Notably, the proposed method detects the fault more quickly. Simultaneous failure of actuators (RW2, 3) occurred between 44 and 55 seconds. As can be seen, the previous method identified only one of the actuators, but the MEMMAE method correctly identified both failures.

A detailed comparison of the test results is provided in Table6.

Table6. The detailed comparison results (Evaluation test)

\begin{tabular}{|c|c|c|c|}
\hline Fault Mode & Features & EMMAE & MEMMAE \\
\hline \multirow{2}{*}{$\begin{array}{c}\text { Fault 1 } \\
14-29\end{array}$} & Responsive time & 17.60 & 14.5 \\
\cline { 2 - 4 } & Faulty actuators & RW1 & RW1 \\
\cline { 2 - 4 } & Cause & - & K \\
\hline \multirow{2}{*}{ Fault 2 } & Responsive time & 45.3 & 44.2 \\
\cline { 2 - 4 } $44-55$ & Faulty actuators & RW3 & RW2-RW3 \\
\cline { 2 - 4 } & Cause & - & K \\
\hline
\end{tabular}


the proposed method has a more responsive speed compare previous, method. In addition, detection fault simulation is a remarkable advantage over the EMMAE method.

\section{Conclusion}

In this paper, different fault detection and diagnosis methods were discussed and due to high reliability, capability to detect faults in the presence of various uncertainties, fast detection process, and the possibility of practical implementation, the Extended Multiple Model Adaptive Estimation (EMMAE) Method were selected. Despite the many capabilities of this method, there were limitations in this method, which the main purpose of this article is to modify. The MEMMAE approach was presented and the steps for its implementation were stated along with the equations. Using this method, limitations of the EMMAE method, including unable to fault diagnostics, simultaneous faults on actuators, and high processing volume in this method were eliminated. The capability of this method was evaluated by performing various tests using MATLAB software and its results compare with the previous method. By conducting various tests, it was determined that the algorithm can detect different faults of the spacecraft actuators in different modes and can be used practically. The study of robustness against disturbances and uncertainties as well as a combination of the EMMAE method with other estimation algorithms will be considered in the next article.

\section{Declarations}

- Availability of data and materials

Up on request.

- Competing interests

We don't have any competing interests to declare.

- $\quad$ Funding

We don't have funding sources to declare.

- Authors' contributions 
In this paper, we report on a model-based fault detection approaches. In this work, modified extended multiple models adaptive estimation (MEMMAE) method is developed which keep both the advantages of the previous model-based methods and take into account some limitations of that.

- Acknowledgements

None

\section{References}

1. Rolf Isermann, Model-based fault-detection and diagnosis status and applications, Annual Reviews in Control, Volume 29, Issue 1,2005, Pages 71-85, ISSN 1367-5788,

2. Katipamula, Srinivas \& Brambley, Michael. (2005). Methods for Fault Detection, Diagnostics and Prognostics for Building Systems - A Review Part I. HVAC and R Research. 11. 10.1080/10789669.2005.10391133.

3. X. Yu, J. Jiang, A survey of fault-tolerant controllers based on safety-related issues, Annual Reviews in Control 39 (2015) 46-57.

4. Johan Carvajal-Godinez, Jian Guo, Eberhard Gill, Agent-based algorithm for fault detection and recovery of gyroscope's drift in small satellite missions,Acta Astronautica,Volume 139,2017, Pages 181-188

5. S. Anwar, W. Niu, A nonlinear observer based analytical redundancy for predictive fault tolerant control of a steer-by-wire system, Asian Journal of Control 16 (2) (2014) 321-334.

6. Said, M., Abdellafou, K.b. \& Taouali, O. Machine learning technique for data-driven fault detection of nonlinear processes. J Intell Manuf 31, 865-884 (2020).

7. T. Gao, W. Sheng, M. Zhou, B. Fang, L. Zheng, "MEMS Inertial Sensor Fault Diagnosis Using a CNN-Based Data-Driven Method", International Journal of Pattern Recognition and Artificial Intelligence, p. 2059048, 2020 
8. H.A. Talebi, and K. Khorasani, "A neural network-based actuator gain fault detection and isolation strategy for nonlinear systems," IEEE Conference on Decision and Control, vol. 46, pp. 2614-2619, 2007

9. Virtic, M., Abersek, B., Zuperl, U. (2008). Using of acoustic models in mechanical diagnostics. Strojniški vestnik - Journal of Mechanical Engineering, vol. 54, no. 12, p. 874882

10. W. Yulei, Y. Jingxin and C. Hong, "Data-driven fault detection for vehicle lateral dynamics," 2017 36th Chinese Control Conference (CCC), Dalian, 2017, pp. 7269-7274, doi: 10.23919/ChiCC.2017.8028504.

11. Belsak, A., Flasker, J. (2008). Vibration analysis to determine the condition of gear units. Strojniški vestnik - Journal of Mechanical Engineering, vol. 54, no. 1, p. 11-24

12. Capozzoli, A., F. Lauro, and I. Khan. 2015. "Fault Detection Analysis Using Data Mining Techniques for a Cluster of Smart Office Buildings." Expert Systems with Applications. 42: 4324-4338.

13. E. Khalastchi and M. Kalech. A sensor-based approach for fault detection and diagnosis for robotic systems. Autonomous Robots, 42(6):1231-1248, Aug. 2018.

14. Cho, Seongpil; Gao, Zhen; Moan, Torgeir. Model-based fault detection of blade pitch system in floating wind turbines. Journal of Physics: Conference Series 2016 ;Volum 753

15. Skliros, C., Esperon Miguez, M., Fakhre, A., Jennions, I. (2019). A review of model based and data driven methods targeting hardware systems diagnostics. Diagnostyka, 20(1), 321. https://doi.org/10.29354/diag/99603

16. Bouallègue,W.; Bouslama Bouabdallah, S.; Tagina, M. Causal approaches and fuzzy logic in FDI of Bond Graph uncertain parameters systems. In Proceedings of the IEEE International Conference on Communications, Computing and Control Applications (CCCA), Hammamet, Tunisia, 3-5 March 2011

17. Thirumarimurugan, M.; Bagyalakshmi, N.; Paarkavi, P. Comparison of fault detection and isolation methods: A review. In Proceedings of the 2016 10th International Conference on Intelligent Systems and Control (ISCO), Coimbatore, India, 7-8 January 2016.

18. Zhao, X.; Zhang, L.; Ouyang, D.; Jiao, Y. Deriving all minimal consistency-based diagnosis sets using SAT solvers. Prog. Nat. Sci. 2009, 19, 489-494.

19. Venkatasubramanian, V.; Rengaswamy, R.; Yin, K.; Kavuri, S. A review of process fault detection and diagnosis: Part I: Quantitative model-based methods. Comput. Chem. Eng. 2003, 27, 293-311.

20. Li, Xifeng \& Xie, Yongle \& Bi, Dongjie \& Ao, Yongcai. (2013). Kalman Filter Based Method for Fault Diagnosis of Analog Circuits. Metrology and Measurement Systems. 20. 10.2478/mms-2013-0027.

21. Geliel, Mostafa \& Zakzouk, Sherief \& Sengaby, M.. (2012). Application of model based fault detection for an industrial boiler. 2012 20th Mediterranean Conference on Control and Automation, MED 2012 - Conference Proceedings. 98-103. 10.1109/MED.2012.6265621.

22. Shim, D.; Yang, C. Optimal Configuration of Redundant Inertial Sensors for Navigation and FDI Performance. Sensors 2010, 10, 6497-6512 
23. Patton, R.; Uppal, F.; Simani, S.; Polle, B. Robust FDI applied to thruster faults of a satellite system. Control Eng. Pract. 2010, 18, 1093-1109.

24. Falcoz, A.; Henry, D.; Zolghadri, A. Robust Fault Diagnosis for Atmospheric Reentry Vehicles: A Case Study. IEEE Trans. Syst. Man Cybern. Part A Syst. Hum. 2010, 40, 886899.

25. T Kobayashi, DL Simon, Hybrid Kalman filter approach for aircraft engine in-flight diagnostics: sensor fault detection case. J. Eng. Gas Turbines Power. 129(3), 746-754 (2006). doi:10.1115/1.2718572.

26. D. Ward, R. L. Barron, M. P. Carley, and T. J. Curtis. Real-time Parameter Identification for Self-designing Flight Control. In Proceedings of the National Aerospace and Electronics Conference (NAECON), Dayton, OH, 1994.

27. D. G. Ward, J. F. Monaco, and M. Bodson. Development and Flight Testing of a Parameter Identification Algorithm for Reconfigurable Control.AIAA Journal of Guidance, Control, and Dynamics, 21(6):948-956, Nov-Dec 1998

28. R. Isermann. Fault-Diagnosis Systems, An Introduction from Fault Detection to Fault Tolerance. Springer-Verlag, Berlin Heidelberg, 2006

29. L. Ni.Fault-Tolerant Control of Unmanned Underwater Vehicles.PhDthesis, VA Tech. Univ., Blacksburg, VA, 2001.

30. M. Azam, K. Pattipati, J. Allanach, S. Poll, and A. Petterson-Hine. In-Flight Fault Detection and Isolation in Aircraft Flight Control Systems. InProceedings of IEEE Aerospace Conference, 2005. Paper 1429.

31. G. Ducard and H. P. Geering, "A reconfigurable flight control system based on the EMMAE method," 2006 American Control Conference, Minneapolis, MN, 2006, pp. 6 pp.-, doi: 10.1109/ACC.2006.1657599.

32. Alikhani Alireza and Sharifi Ghasem, "Application of a Model-Based Fault Detection Approach on a Spacecraft", International Journal of Reliability, Risk and Safety: Theory and Application, Vol. 2, 2020.

33. Moradi, Rouzbeh \& Alikhani, Alireza \& Fathi, Mohsen. (2017). Reducing the Effects of Inaccurate Fault Estimation in Spacecraft Stabilization. Journal of aerospace technology and management. 9. 10.5028/jatm.v9i4.826.

34. Marcel Sidi, "Spacecraft Dynamics \& Control," Cambridge university press, 1997

35. R. Cowen, "The wheels come off Kepler," Nature, vol. 497, no. 7450, pp. 417-418, May 2013.

36. S. Murugesan and P. S. Goel, "Fault-tolerant spacecraft attitude control system," Sadhana, vol. 11, no. 1-2, pp. 233-261, Oct. 1987.

37. Rahimi, Afshin. (2017). Fault Diagnosis and Prognosis of Satellite Attitude Control System with Reaction Wheels and Control Moment Gyros.

38. Ghasem Sharifi \& Ehsan Zabihian (2020) An effective approach to identify the mass properties of a satellite attitude dynamics simulator, Australian Journal of Mechanical Engineering, 18:3, 245-254, DOI: 10.1080/14484846.2018.1458811

39. V. Carrara, "Comparison between means of attitude control with reaction wheels," in Proceedings of the 6th Brazilian Symposium on Inertial Engineering (SBEIN '10), Rio de Janeiro, Brazil, November 2010. 
40. Ousaloo HS, Sharifi G, Akbarinia B. Extended validation of a ground-based three-axis spacecraft simulator model. Proceedings of the Institution of Mechanical Engineers, Part G: Journal of Aerospace Engineering. 2021;235(2):151-170.

41. Jung, D. and P. Tsiotras. A 3-dof experimental test-bed for integrated attitude dynamics and control research. in AIAA Guidance, Navigation and Control Conference. 2003.

42. Simon Haykin, Adaptive Filter Theory, Prentice Hall, 2002, ISBN 0-13-048434-2

43. Ducard, G.. (2009). Fault-tolerant Flight Control and Guidance Systems: Practical Methods for Small Unmanned Aerial Vehicles. 10.1007/978-1-84882-561-1.

44. M.S. Grewall, A.P.Andrews. Kalman Filtering. Theory and Practice. Prentice Hall, 1993

45. D. Rupp, G. Ducard, H. P. Geering, and E. Shafai. Extended Multiple Model Adaptive Estimation for the Detection of Sensor and Actuator Faults. InProceedings of IEEE Control and Decision Conference, and European Control Conference, pages 3079-3084, Seville, Spain, 2005.

46. R. H. Chen and J. L. Speyer. Sensor and Actuator Fault Reconstruction.AIAA Journal of Guidance, Control, and Dynamics, 27(2):186-196, March-April 2004. 